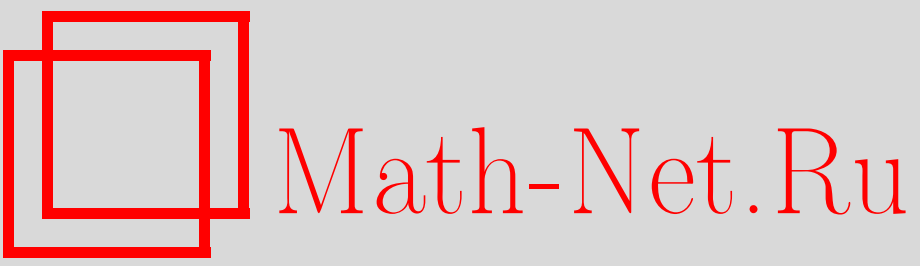

В. В. Зудилин, Об иррациональности значений дзета-функции в нечетных точках, УМH, 2001, том 56, выпуск 2, 215-216

DOI: https://doi.org/10.4213/rm389

Использование Общероссийского математического портала Math-Net.Ru подразумевает, что вы прочитали и согласны с пользовательским соглашением

http://www.mathnet.ru/rus/agreement

Параметры загрузки:

IP: 52.6 .47 .48

26 апреля 2023 г., 17:35:38 


\title{
ОБ ИРРАЦИОНАЛЬНОСТИ ЗНАЧЕНИЙ ДЗЕТА-ФУНКЦИИ В НЕЧЕТНЫХ TOЧКАХ
}

\author{
В.В. Зудилин
}

В настоящее время известно лишь несколько результатов об арифметической природе значений дзета-функции Римана $\zeta(s)$ в нечетных точках $s>1$. Первый из них - иррациональность $\zeta(3)$ - получил Р. Апери в 1978 г. [1], а последний принадлежит Т. Ривоалю [2], доказавшему в 2000 г. асимптотическую оценку размерности $\delta(a)$ пространств, натянутых над $\mathbb{Q}$ на числа $1, \zeta(3), \zeta(5), \ldots, \zeta(a-2), \zeta(a)$, где $a$ нечетно:

$$
\delta(a) \geqslant \frac{\log a}{1+\log 2}(1+o(1)) \quad \text { при } a \rightarrow \infty .
$$

В частности, из (1) следует бесконечность множества иррациональных чисел среди $\zeta(3), \zeta(5), \ldots$ Обобщая конструкцию Ривоаля [2], мы доказываем следующие резулттаты.

Tеорема 1. В каждом числовом наборе

$$
\begin{gathered}
\{\zeta(5), \zeta(7), \zeta(9), \zeta(11), \zeta(13), \zeta(15), \zeta(17), \zeta(19), \zeta(21)\}, \\
\{\zeta(7), \zeta(9), \zeta(11), \ldots, \zeta(35), \zeta(37)\} \\
\{\zeta(9), \zeta(11), \zeta(13), \ldots, \zeta(51), \zeta(53)\}
\end{gathered}
$$

имеется по крайней мере одно иррачиональное число.

Теорема 2. Существует нечетное число $a \leqslant 145$ такое, что числа $1, \zeta(3), \zeta($ (а) линейно независимы над $\mathbb{Q}$.

Теорема 2 усиливает соответствующий резултат работы [3]: $a \leqslant 145$ вместо $a \leqslant 169$.

Теорема 3. Для каждого нечетного $а \geqslant 3$ справедлива абсолютная оценка

$$
\delta(a)>0.395 \log a>\frac{2}{3} \cdot \frac{\log a}{1+\log 2} .
$$

Зафиксируем положительные нечетные параметры $a, b, c$, причем $a>b(c-1)$ и $c \geqslant 3$, и для каждого положительного $n$ рассмотрим рациональную функцию

$$
\begin{aligned}
R(t) & =R_{n}(t):=\frac{((t \pm(n+1)) \cdots(t \pm c n))^{b}}{(t(t \pm 1) \cdots(t \pm n))^{a}} \cdot(2 n) !^{a+b-b c} \\
& =(-1)^{n}\left(\frac{\Gamma( \pm t+c n+1)}{\Gamma( \pm t+n+1)}\right)^{b} \cdot\left(\frac{\Gamma(t) \Gamma(1-t)}{\Gamma( \pm t+n+1)}\right)^{a} \cdot(2 n) !^{a+b-b c},
\end{aligned}
$$

где знак ' \pm ' означает, что в соответствующем произведении появляются множители, отвечающие как знаку ' + ', так и '-'. Представляя функцию (3) в виде суммы простейших дробей и используя ее нечетность и поведение при $t \rightarrow \infty$, заключаем, что

$$
I=I_{n}:=\sum_{t=n+1}^{\infty} \frac{1}{(b-1) !} \frac{\mathrm{d}^{b-1} R(t)}{\mathrm{d} t^{b-1}}=\sum_{\substack{s \text { нечетно } \\ b<s<a+b}} A_{s} \zeta(s)-A_{0},
$$

где коэффициенты $A_{s}=A_{s, n}$ линейной формы $I$ - некоторые рациональные числа. (При $b=1$, $c=2 r+1$ мы получаем те же линейные формы (4), что и в работе [2].) Обозначим через $D_{n}$ наименьшее общее кратное чисел $1,2, \ldots, n$; при этом, как хорошо известно,

$$
\lim _{n \rightarrow \infty} \frac{\log D_{n}}{n}=1 .
$$

Работа выполнена при частичной поддержке фонда INTAS и Российского фонда фундаментальных исследований (грант № IR-97-1904). 
Лемма 1. Для каждого нечетного $c \geqslant 3$ существует последовательность цельх $\Pi_{n}=\Pi_{n}^{(c)} \geqslant 1, n=1,2, \ldots$, такая, что числа $\Pi_{n}^{-b} D_{2 n}^{a+b-1} A_{s, n}$ являются чельмии и справедливо предельное соотношение

$$
\varpi_{c}:=\lim _{n \rightarrow \infty} \frac{\log \Pi_{n}^{(c)}}{n}=-\sum_{l=1}^{(c-1) / 2}\left(2 \psi\left(\frac{2 l}{c-1}\right)+2 \psi\left(\frac{2 l}{c}\right)+\frac{2 c-1}{l}\right)+2(c-1)(1-\gamma),
$$

где $\gamma \approx 0.57712$ - постоянная Эйлера, а $\psi(x)$ - логарифмическая производная гаммафункции. (При с $\rightarrow \infty$ величина $\varpi_{c}$ в (5) имеет порядок $2 c(1-\gamma)+O(\log c)$.)

Лемма 1 усиливает соответствующие оценки знаменателей линейных форм (4) за счет появления множителя $\Pi_{n}^{-b}$ даже в случае $b=1$, рассмотренном в работе [2]. Это обстоятельство является ключевым при выводе теорем 2,3 .

Доказательство следующего утверждения опирается на представление форм (4) в виде контурных интегралов и применение метода перевала (ср. с [4], [5]). В случае $b>1$ на параметры $a, b, c$ накладываются дополнительные ограничения, которые оказываются автоматически выполненными в приложении к теореме 1 .

ЛЕмма 2. Для линейных форм (4) справедливо предельное соотношение

$$
\varkappa:=\varlimsup_{n \rightarrow \infty} \frac{\log \left|I_{n}\right|}{n}=\log \frac{2^{2(a+b-b c)}\left|\tau_{0}+c\right|^{b c}\left|\tau_{0}-c\right|^{b c}}{\left|\tau_{0}+1\right|^{a+b}\left|\tau_{0}-1\right|^{a+b}}
$$

где $\tau_{0}$ - вещественный корень многочлена $(\tau+c)^{b}(\tau-1)^{a+b}-(\tau-c)^{b}(\tau+1)^{a+b}$ из интервала $(c,+\infty)$ в случае $b=1$ и один из пары комплексно-сопряженных корней с максимально возможной частью $\operatorname{Re} \tau_{0}$ в случае $b>1$. (Для $b=1$ верхний пре-

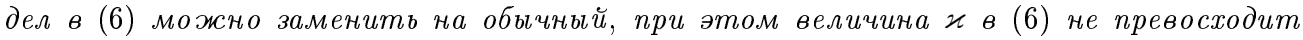
$(2 a-c+3) \log 2-2(a-c+1) \log c$.

Согласно леммам 1,2 в случае $-b \varpi_{c}+2(a+b-1)+\varkappa<0$ среди чисел $\zeta(s)$, где $s$ нечетно и $b<s<a+b$, имеется по крайней мере одно иррациональное. Выбирая $a=19, b=3, c=3$; $a=33, b=5, c=3$ и $a=47, b=7, c=3$ соответственно для каждого из наборов в (2), получаем теорему 1 .

Лемма 3. Для ненулевых коэффичиентов $A_{s}=A_{s, n}$ линейных форм (4) справедлива оценка

$$
\varlimsup_{n \rightarrow \infty} \frac{\log \left|A_{s, n}\right|}{n} \leqslant 2 b c \log c+2(a+b-b c) \log 2 .
$$

Теоремш 2,3 вьводятся из лемм 1-3 с помощью критерия линейной независимости из [6] так же, как и в [2]. Для доказательства теоремы $2(a=145, b=1)$ мы выбираем $c=21$.

\section{СПИСОК ЛИТЕРАТУРЫ}

[1] R. Apéry // Astérisque. 1979. V. 61. P. 11-13. [2] T. Rivoal // C. R. Acad. Sci. Paris. Sér. I Math. 2000. V. 331. № 4. P. 267-270. [3] T. Rivoal // Rapport de recherche SDAD № 2000-9. Univ. Caen, 2000. [4] Ю. В. Нестеренко // Матем. заметки. 1996. Т. 59. №6. С. 865-880. [5] Т. Г. Хессами Пилеруд. Арифметические свойства значений гипергеометрических функций // Дисс. ... канд. физ.-матем. наук. М.: МГУ, 1999. [6] Ю.В.Нестеренко // Вестник МГУ. Сер. 1. Матем., мех. 1985. № 1. С. 46-54. 